\title{
Tele-Healthcare with Humanoid Robots: A User Study on the Evaluation of Force Feedback Effects
}

\author{
Michael Panzirsch, Bernhard Weber, Luis Rubio, Sofia Coloma, Manuel Ferre and Jordi Artigas
}

\begin{abstract}
The progress of robotic technology in the last years paved the way for its application in the healthcare sector. In the light of demographic change and lack of nursing staff, telerobotic systems are a promising solution e.g. for homecare in rural areas or in quarantine stations to prevent dissemination of pathogens. This paper presents a user study investigating human performance when teleoperating a complex humanoid robot with an underactuated haptic interface. Twelve novices had to complete basic movement and contact tasks as well as more typical healthcare tasks like instrument handling and usage (pulse oximetry). Besides the general performance level we explored the effect of different force feedback architectures (with vs. without sensor-based force information) and of workspace scaling (1:1 vs. $1: 2)$. The test subjects showed in the majority of tasks significantly best performance with the 3-Channel architecture and a scaling of 1:2.
\end{abstract}

Index Terms-Teleoperation, Tele-Healthcare, Workspace Scaling, 3-Channel

\section{INTRODUCTION}

The Ebola outbreak in West Africa in 2014 agitated far more people than were directly impacted. Not only volunteering healthcare professionals but also medical staff in European hospitals were threatened by contagion. Though technology that is able to reduce the risk of contamination is already used in other harmful areas like in nuclear power plants or the offshore industry, it did not find the way into quarantine facilities yet.

In comparison to surgical applications, robots that are meant to support or even to some extent replace nurses have to accomplish other requirements. Still, the required level of reliability is similar since healthcare robots may directly interact with the patient. High adaptability to different tasks, high maneuverability and the adequate interaction with the patient need to be considered. According to [1], physicians require that teleoperation systems provide permanent and full information e.g. on temperature, pulse and blood pressure similar to the general working environment in a hospital. In addition, the different communication channels for haptic, visual and auditive feedback need to be perfectly synchronized.

A large number of healthcare robots have been developed for similar fields of application [2]. The robot Cody is able to wash human limbs autonomously and an evaluation of the patient's system showed that acceptance strongly depends on the interpretation of robot intention [3]. Other robots serve the rehabilitation [4], the nurse assisting lifting of patients [5] or even blood sampling [6].

In contrast to these systems, we propose a versatile telepresence system allowing to perform a wide range of healthcare activities remotely i.e. no staff has to be at the patient's site. As a first step of evaluation, the present user study investigates the performance level of inexperienced subjects without healthcare background during elementary tasks (robot positioning) and basic procedures (grasping, tool interaction) that are involved in any tele-healthcare activity. The main objective was to set up a system that can be used intuitively by novices. The intuitiveness depends e.g. on network characteristics, workspace mapping, field of view, quality of haptic feedback and so forth [7]. Here, we focused on the impact of the haptic feedback realism and workspace mapping on operators' performance.

In literature, the indispensability of force feedback in general is clearly stated [8]. In a medical scenario, the authors of [9] found that force feedback can reduce the task completion time and improve the accuracy of surgeons that have a high cognitive load. During complex and delicate telesurgical tasks, force feedback is crucial to adjust the input forces adequately and to avoid exaggerated forces (e.g. damaging tissue, breaking threads).

The evaluation of the necessity of torque feedback in [10] is of high importance concerning the application of a device that enables only force feedback in this study. Similar to the results of [11] it was shown that force only feedback can approximate the feedback of force and torque though transparency (quality of immersion of the human operator into the slave robot's environment) is always reduced for underactuated devices that do not provide torque feedback. Still, [12] suggests that the use of input devices with $6 \mathrm{DoF}$ compared to $3 \mathrm{DoF}$ force feedback leads to significantly better user perception and performance.

It was shown e.g. in [13] that a combination of computed and measured force feedback in a 3-Channel approach leads to higher fidelity compared to systems with pure computed or measured force feedback. The authors of [14] showed that in theory a class of 3-Channel architectures can lead to perfect transparency. Since most low-cost haptic devices have no force sensor, a 4-Channel architecture [15] can not be implemented in the analyzed setup. Since the 3-Channel 


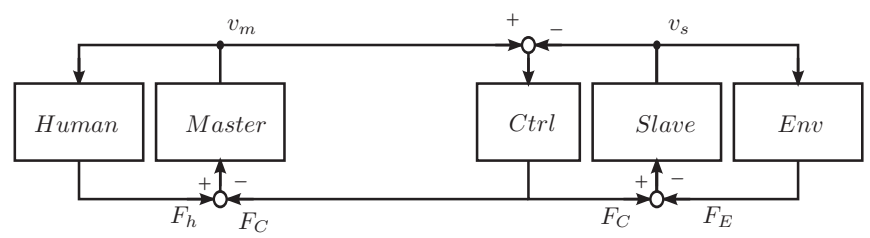

Fig. 1. 2-Channel Architecture with Computed Force Feedback

architecture has advantages compared to a 2-Channel architecture and measured force-feedback requires a costly forcetorque sensor at the slave side, the 3-Channel architecture (compare Fig. 2) is compared with a 2-Channel architecture with pure computed force feedback (see Fig. 1). In the 3Channel architecture, the computed force $F_{C}$ is scaled down by $\lambda_{C}$ and the measured force is scaled down by $\lambda_{E}$.

The second variable considered here is the workspace scaling of master and slave device as the optimal scaling strongly depends on the system design and the chosen task [16]. In general, a 1:1 mapping of master and slave motion should lead to higher movement accuracy compared to a upscaled (1:2) motion mapping. Still, the larger movement amplitudes in the 1:1 mapping may also lead to decreased performance in slow, high-precision movements, because hand/arm stabilization is more demanding.

When working with a 2-Channel architecture based on calculated force values the inertia of the telemanipulated robot will be conveyed to the user and should have an impact on performance. Compared to the 3-Channel architecture additionally based on measured forces where this inertia effect should be greatly reduced, the telemanipulation performance should be decreased when quick movements (like aiming or corrective motions) are required. Moreover, the transparency of the system should decrease in the 2Channel case, leading to attenuated accuracy when regulating interaction forces and workload should increase. Yet, to a certain degree inertia might even be beneficial when slow and smooth movements have to be performed (like slow tracking tasks). When working with a measured compared to a pure calculated force approach:

H1: Task completion times should be shorter

H2: Movement accuracy should be higher during fast movements

H3: Contact forces during robot-environment interactions should be lower

H4: Subjective contact realism should be higher and thus mental workload lower

We explored whether these effects are further moderated by scaling (1:1 vs 1:2).

In section 2 the technical and experimental setup is explained. The results of the user study are evaluated in section 3. The discussion of the results and the conclusion on the system design are presented in section 4 .

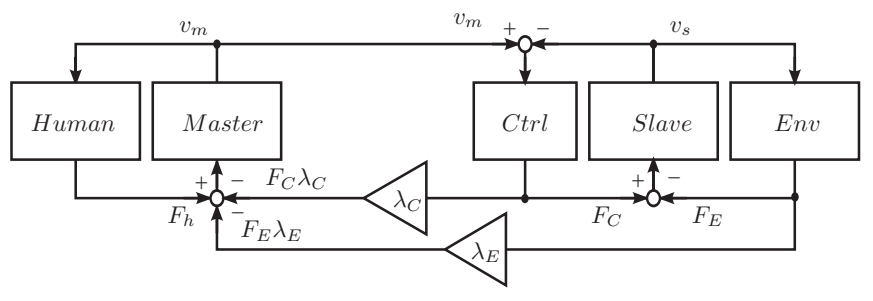

Fig. 2. 3-Channel Architecture

\section{METHOD}

\section{A. Participants}

The user study was conducted with $\mathrm{N}=12$ participants ( 9 male, 3 female) with an age between 22 and 35 years $(\mathrm{M}=25,83 ; \mathrm{SD}=4,24)$. The sensorimotor performance of every test subject was measured via the "Sensomotorische Koordination" test battery (SMK, [17]) a part of the Vienna Test System of the Schuhfried GmbH. The SMK data $\left(M_{S M K}=74,42\right.$; $S D_{S M K}=14,29$; Range: $\left.45-93\right)$ were normally distributed as indicated by a non-significant ShapiroWilks-Test (W $=.941 ; \mathrm{p}=.508 ;[18])$.

\section{B. Technical Setup}

The humanoid robot SpaceJustin (see Fig. 3) was applied as the slave robot in the presented user study. The two impedance controlled slave arms are DLR light weight robots (LWR) and equipped with a DLR HitHand II each. The head can be moved in two degrees of freedom and is equipped with two Prosilica cameras. The master console (see Fig. 4) consisted of two Phantom devices (Phantom Omni, Sensable Technology, Woburn, MA) and the head mounted display (HMD) Oculus Rift DK2 developer edition. The HMD displayed the 3D video stream from SpaceJustin's cameras. A pedal was used as a deadman switch that controls the coupling to the slave robotic system. Via the buttons of the Phantom device the opening and closing of the robotic hand could be triggered. The workspace center of the haptic device was marked by a piece of fabric on the table to provide a reference position even while wearing the HMD. A Xsens inertial measurement unit was fixed to the head mounted display and allowed the control of the two respective degrees of freedom of the head.

The controller of the haptic device was running on a Linux system and programmed in ROS. The slave robot and hand controller were implemented in Matlab Simulink and running on a RT Linux system. The master and slave were communicating via UDP in a local network such that time delay was negligible. The relative pose of the master device (referring to the initial state) was sent to the slave device in an interface with a position vector and unit quaternions. A spatial spring was implemented to penalize a position deviation of master and slave device. When the pedal of the master was released the last commanded slave pose was maintained in order to freeze the slave's pose. Thus, an 


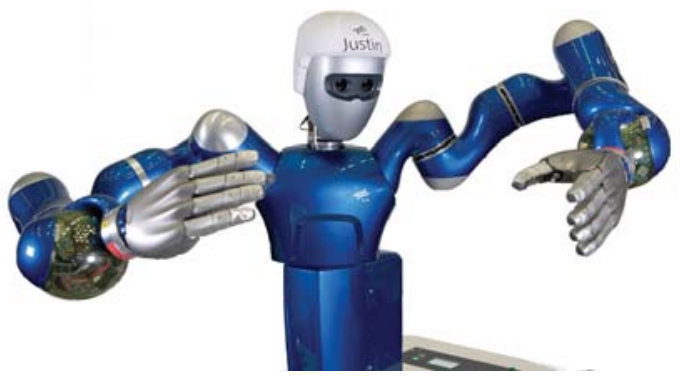

Fig. 3. DLR SpaceJustin

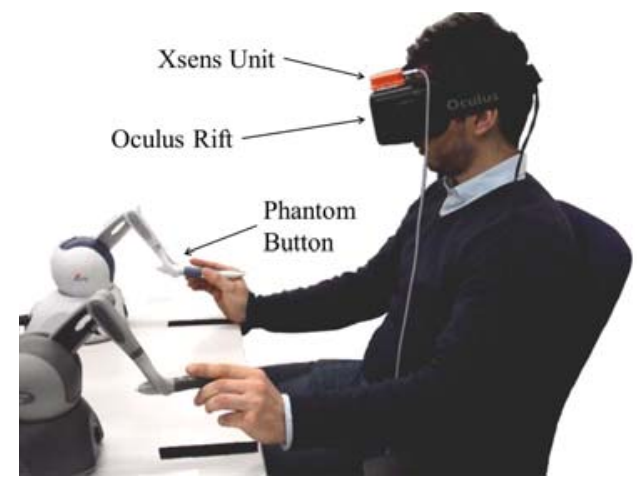

Fig. 4. Master Console with Phantom Omni, Oculus and Xsens Unit

indexing method [19] was realized to extend the workspace in an iterative manner.

The Phantom device which has been developed for the interaction in virtual environments [20] has already been applied to several general teleoperation applications and also in medical scenarios. The Phantom has e.g. been employed for the evaluation of the compact modular robot system for laparoscopic surgery presented in [21] and in a virtual palpation training scenario [22].

The LWR has been used in several medical scenarios. The authors of [23] proposed two control methods for a LWR in an ultrasonic examination system. Besides a collaborative teleoperation mode a semi-automatic mode was presented in which the robot drives autonomously to a chosen location maintaining a constant interaction force with the body. In a similar setup with the LWR the authors of [24] implemented an autonomous system for ultrasonic examination controlled via a learning from demonstration approach. Even the prototype robotic system in [25] for minimal invasive surgery is based on this technology.

\section{Tasks}

Three task sets for accuracy analysis, grasping performance and pulse oximetry were considered in the user study design. In the first set a grasping adapter equipped with a pen (compare Fig. 5) was grasped with the right robotic hand. During the Aiming task, subjects had to point at the

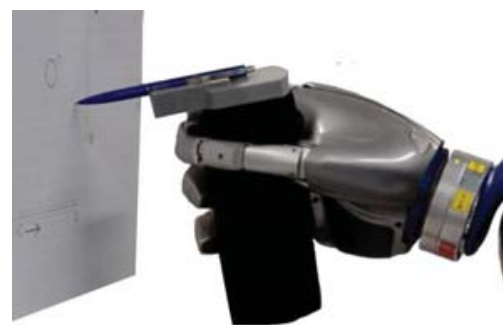

Fig. 5. Grasping Adapter with Pen

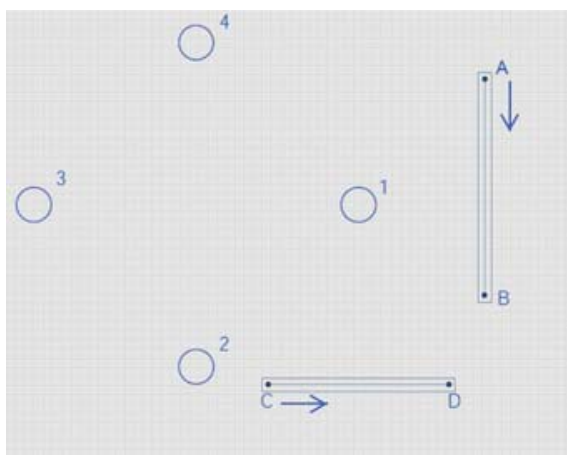

Fig. 6. Millimeter Graph Paper

middle of four different circles ( $2 \mathrm{~cm}$ diameters) as accurately as possible. During the Line Drawing task, a vertical line (see Fig. 6, $\mathrm{A} \rightarrow \mathrm{B})$ and then a horizontal line $(\mathrm{C} \rightarrow \mathrm{D}$, both $11 \mathrm{~cm}$ ) had to be drawn. This newly developed task allows the direct accuracy measurement (Aiming and Line Drawing) without the necessity to track the tool tip motion with respect to the desired motion via stereo vision or tracking modules.

The third task was the Grasping of a tool adapter equipped with an oximetry unit (see Fig 7). The test subject had to approach the object and trigger the grasp via the Phantom button. After lifting, the object had to be put on the table and released again. In the fourth task (Oximetry), the oximetry unit had to be plugged onto an artificial finger (see Fig. 8 ). Then the activation button (diameter of $3 \mathrm{~mm}$ ) had to be pressed with the left robotic hand. After this, the unit had to be removed from the finger. The adapter was already grasped
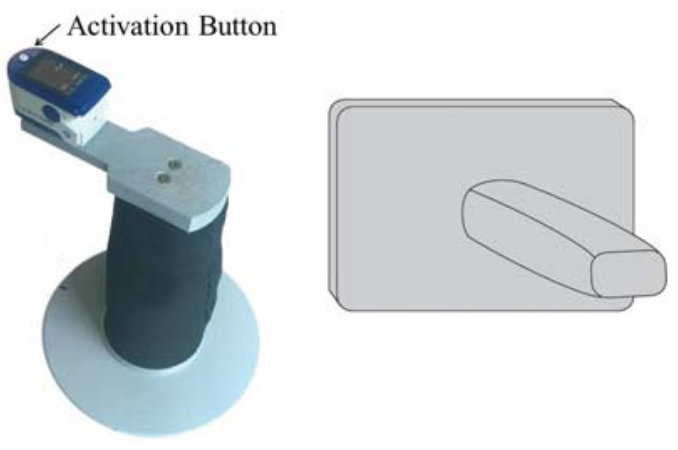

Fig. 7. Oximetry Unit Fig. 8. Finger Dummy 
in the initial state of this task. For each task a specific initial position of the robotic arms was chosen.

\section{Experimental Design and Procedure}

In the presented user study, four conditions were compared. A 2-Channel architecture with computed force feedback and a 3-Channel architecture with combined computed and measured force feedback was implemented. The scaling was varied such that in a low scaling condition the unaltered master motion was transmitted as the desired slave motion. The high scaling doubled the slave motion with respect to the master motion. This resulted in four conditions:

- Computed force feedback, 1:1 workspace scaling $(2 \mathrm{Ch} / \mathrm{Sc} 1)$

- Computed force feedback, 1:2 workspace scaling $(2 \mathrm{Ch} / \mathrm{Sc} 2)$

- Computed and measured force feedback, 1:1 workspace scaling $(3 \mathrm{Ch} / \mathrm{Sc} 1)$

- Computed and measured force feedback, 1:2 workspace scaling $(3 \mathrm{Ch} / \mathrm{Sc} 2)$

The scaling $\lambda_{C}$ and $\lambda_{E}$ had a ratio of $\frac{\lambda_{C}}{\lambda_{E}}=\frac{0.3}{0.7}$. A withinsubject 2(Channel)*2(Scaling) design has been implemented i.e. each subject completed all four conditions in systematically varied order to control for time effects like fatigue or training. For the Line Drawing task a fifth condition was implemented in which the task was performed manually without the telerobotic system. Within each condition all tasks were performed in the following order: 1) Aiming, 2) Line Drawing, 3) Grasping and 4) Oximetry. Prior to each set of tasks, subjects performed a test trial to become familiar with the task demands.

After each condition (four times for each task set) the contact realism and the overall workload scale [26] was rated (scale range from 1 to 20 ).

\section{E. Data Analysis}

The slave software logged the duration, the desired and actual position, the velocity and the computed and measured interaction forces and torques of the slave. This data was evaluated with Matlab 2013b.

\section{RESUlts}

All descriptive results (means and standard deviations) are presented in Table I. Data were analyzed performing repeated measures ANOVA (rmANOVA). The main assumptions for this procedure i.e. normality of residuals and sphericity were tested by Shapiro-Wilk's test and Mauchly's test. Only violations of these assumptions are reported in the following analyzes. In the case of non-normality the non-parametric, related-samples Friedman test as global test and Wilcoxon signed rank tests for pairwise comparisons were conducted.

Task Duration First, we analyzed the task durations for all of the four tasks. For Aiming, the assumption of normality was violated and thus the Friedman test was chosen. Results indicated a significant effect of conditions $(p<.05)$. Subsequent Wilcoxon signed rank tests revealed that there is a significant scaling effect on task durations in the 2Channel as well as in the 3-Channel conditions (both ps $<.05$ ), i.e. subjects were substantially faster with the 1:2 scaling. Moreover, we found at least a trend that with 1:2 scaling subjects needed less time in the 3-Channel compared to the 2-Channel condition ( $\mathrm{p}<.10$; one-tailed testing). In the Line Drawing task normality was not given. Friedman analysis also showed an overall condition effect $(p<.001)$ and the same scaling effect in the 2-Channel as well as in the 3-Channel conditions (both ps <.05). Moreover, subjects performed significantly faster with the 3-Channel architecture in the $1: 2$ scaling conditions ( $p<.05$, one-tailed testing) and marginally faster in the $1: 1$ scaling conditions $(p<.10$, onetailed testing). No significant condition effect was evident for the Grasping task as indicated by the Friedman test. Finally a marginally significant condition effect was found for the Oximetry task $(\mathrm{p}<.10)$, with shorter times in the 3 Channel compared to the 2-Channel conditions as indicated by Wilcoxon tests $(\mathrm{p}<.10)$. Yet this effect only occurred in the $1: 2$ scaling conditions.

Force Application We analyzed the forces during Line Drawing along the $\mathrm{x}$-dimension (depth). rmANOVA indicated a significant channel main effect $(\mathrm{F}(1,11)=8.0, \mathrm{p}=$ $.01)$ and a marginal scaling $\mathrm{x}$ Channel interaction $(\mathrm{F}(1,11)$ $=3.2, \mathrm{p}=.10)$. Hence, forces were substantially lower in the 3-Channel case and this effect was particularly pronounced in the 1:1 scaling condition. A subsequent paired-samples T-test also confirmed the significantly lower forces with 3 Channel during $1: 1$ scaling $(\mathrm{t}(11)=2.93 ; \mathrm{p}=.01)$ and at least a trend for the same effect during 1:2 scaling $(\mathrm{t}(11)=$ $1.59 ; \mathrm{p}<.10$, one-tailed testing).

Positional Accuracy for Aiming and Line Drawing was explored next. The accuracy measure for Aiming was the distance between the aim and the subjects' point on the sheet. A rmANOVA was performed and revealed a significant effect of scaling $(\mathrm{F}(1,11)=5.0, \mathrm{p}<.05)$ with higher average accuracy in the 1:2 scaling conditions. Subsequent T-tests indicated that the scaling effect was exclusively due to the 3-Channel conditions $(\mathrm{t}(11)=2.21, \mathrm{p}<.05)$, with a relatively large distance of $\mathrm{M}=1.48 \mathrm{~mm}$ during $1: 1$ scaling and significantly smaller distances of $\mathrm{M}=0.81 \mathrm{~mm}$ in the 1:2 scaling condition. No such effect was found for the 2Channel conditions $(\mathrm{t}(11)=0$, ns. $)$.

The main performance measure for the Line Drawing task was the average deviation from the ideal line. In a graphical analysis, the minimum and maximum distances from the ideal line were determined for each of the 10 centimeter intervals along the ideal line. Then, each minimum and maximum value was averaged and the mean across all of the ten intervals was calculated, as general accuracy measure. Here a manual performance baseline was available. Data were non-normal and hence Friedman test was performed, showing a highly significant effect $(\mathrm{p}<.001)$. First of all, accuracy decreased substantially with five times higher deviations in the four telerobotic conditions $(\mathrm{M}=1.13)$ compared 


\begin{tabular}{|c|c|c|c|c|c|c|}
\hline Measure & Unit & $2 \mathrm{Ch} / \mathrm{Sc} 1$ & $3 \mathrm{Ch} / \mathrm{Sc} 1$ & $2 \mathrm{Ch} / \mathrm{Sc} 2$ & $3 \mathrm{Ch} / \mathrm{Sc} 2$ & Manual \\
\hline Task Duration - Aiming & $\sec$ & $60,54(22,68)$ & $63,02(38,83)$ & $54,20(23,20)$ & $50,95(21,96)$ & \\
\hline Task Duration - Line Drawing & $\mathrm{sec}$ & $73,08(40,38)$ & $68,97(40,43)$ & $59,86(29,31)$ & $55,21(33,35)$ & \\
\hline Task Duration - Grasping & $\mathrm{sec}$ & $39,94(11,84)$ & $44,93(18,10)$ & $38,92(5,04)$ & $36,15(13,45)$ & \\
\hline Task Duration - Oximetry & $\sec$ & $35,71(10,10)$ & $32,16(6,58)$ & $39,91(29,46)$ & $27,99(12,26)$ & \\
\hline Force Application (X-Axis) - Line Drawing & $\mathrm{N}$ & $3.58(1.47)$ & $2.86(1.65)$ & $3.64(2.0)$ & $3.35(1.98)$ & \\
\hline Positional Accuracy - Aiming & $\mathrm{mm}$ & $1,15(0,46)$ & $1,48(1,02)$ & $1,15(0,56)$ & $0,81(0,34)$ & \\
\hline Positional Accuracy - Line Drawing & $\mathrm{mm}$ & $1,23(0,48)$ & $1,30(0,43)$ & $0,95(0,31)$ & $1,02(0,29)$ & $0,21(0,21)$ \\
\hline Workload - Aiming \& Line Drawing & $1-20$ & $8.67(4.89)$ & $9.83(4.1)$ & $9.33(4.66)$ & $8.75(4.65)$ & \\
\hline Workload - Grasping & $1-20$ & $7,92(4,12)$ & $8,75(5,56)$ & $7,25(3,86)$ & $7,25(4,92)$ & \\
\hline Workload - Oximetry & $1-20$ & $7.67(3.75)$ & $8.0(4.11)$ & $8.25(4.77)$ & $6.92(4.14)$ & \\
\hline Contact Realism - Aiming \& Line Drawing & $1-20$ & $11.17(4.61)$ & $10.0(4.75)$ & $11.33(4.54)$ & $11.83(4.43)$ & \\
\hline Contact Realism - Grasping & $1-20$ & $9,17(5,01)$ & $10,33(4,87)$ & $10,67(4,81)$ & $11,50(4,50)$ & \\
\hline Contact Realism - Oximetry & $1-20$ & $10.0(5.85)$ & $10.75(4.31)$ & $10.33(5.38)$ & $11.92(3.83)$ & \\
\hline
\end{tabular}

TABLE I

RESULTS

to the manual condition $(\mathrm{M}=0.21)$. The data in the manual condition only were non-normal. Friedman test indicated a highly significant overall effect $(\mathrm{p}<.001)$ and all Wilcoxon tests comparing manual and telerobotic conditions reached significance (all ps <.01). Next rmANOVA was conducted on the data in the telerobotic conditions exclusively and a significant scaling main effect occurred $(\mathrm{F}(1,11)=7.51, \mathrm{p}$ $<.05)$, i.e. accuracy was better with 1:2 compared to $1: 1$ scaling.

Mental Workload Combined subjective ratings for Aiming and Line Drawing indicated that participants perceived lower mental workload when working with 3-Channel in the 1:2 scaling condition (significant rmANOVA Channel x scaling interaction effect, $\mathrm{F}(1,11)=5.0, \mathrm{p}<.05)$. Paired-sampling T-test also revealed a significant difference between 2- vs 3 -Channel in the 1:2 scaling conditions $\left(t_{1: 2}(11)=1.87, \mathrm{p}\right.$ $<.05$, one-tailed testing). For Grasping we found a scaling main effect $(\mathrm{F}(1,11)=9.16, \mathrm{p}<.05)$ with lower ratings in the $1: 2$ compared to the $1: 1$ scaling condition. No significant effects were found for the Oximetry task.

Contact Realism No significant effects were evident for the combined Aiming and Line Drawing ratings regarding contact realism. For the Grasping task there was a significant scaling main effect $(\mathrm{F}(1,11)=8.64, \mathrm{p}=.01)$ and a channel main effect $(\mathrm{F}(1,11)=5.39, \mathrm{p}<.05)$, reflecting higher perceived contact realism with 1:2 scaling as well as 3Channel architecture. Finally, we analyzed the ratings after the Oximetry task, but no significant effects were indicated by rmANOVA.

\section{Discussion}

In the current user study with 12 subjects we explored whether basic movement and contact tasks as well as more realistic tasks from the medical domain can be performed with a complex humanoid robot controlled via a commercially available haptic input device. Nevertheless, movement accuracy was approx. 5 times lower compared to manual performance in the Line Drawing task which is far more delicate than typical healthcare tasks.

We hypothesized that a higher degree of teleoperation transparency and thus performance is reached when implementing a 3-Channel architecture integrating sensor-based force information from the robot. In $\mathrm{H} 1$ we specifically assumed that teleoperation tasks can be accomplished faster. Regarding free movement tasks (Aiming), movements in contact (Line Drawing) and complex tool interaction (Oximetry) we found evidence for shorter completion times with the 3-Channel compared to the 2-Channel approach, although this effect mainly occurred when operation movements were upscaled (factor 1:2). With the upscaling factor, the robot movements were performed faster and thus inertia effects were also more pronounced. For the Grasping task, spatial orientation demands seem to play a more prominent role, hence our experimental conditions did not have an impact. Altogether $\mathrm{H} 1$ is at least partially confirmed.

In $\mathrm{H} 2$ we expected a higher degree of movement accuracy with the 3-Channel solution. In the Aiming task the best performance was achieved with 3-Channel in the 1:2 scaling situation, but also the worst performance in the 1:1 scaling 
condition. Maybe the additional inertia effects in the 2Channel conditions stabilized the larger movement amplitudes in the 1:1 scaling case, leading to better results compared to inertia free movements during 3-Channel conditions. Regarding Line Drawing we did not find any support for our assumption. Perhaps movements were to slow to trigger any effect of Channel architecture. Altogether, H2 is only partially substantiated.

Next, we examined H3, stating that contact forces should be lower when working with the 3-Channel approach. We focused on the Line Drawing task as being the most relevant force regulation task. Here we gathered supportive data for our assumption, i.e. forces were lower with the 3-Channel compared to 2-Channel architecture.

The overall result for the ratings of contact realism and workload showed the best results for the 3-Channel architecture in the 1:2 scaling case. Yet, these did not reach significance in all cases. We only found evidence for higher contact realism with 3-Channels for the Grasping task, with the best results in the 1:2 scaling condition. Also workload was rated best in this condition, but only for the Aiming and Line Drawing tasks. H4 is only valid for a subset of tasks and conditions.

Altogether we found clear advantages of using a control approach integrating measured force feedback regarding relevant performance parameters. Seemingly, the disturbing effects of robot inertia when using a 2-Channel approach deteriorate performance. This effect was only evident when using an upscaling factor (1:2) magnifying the negative impact of inertia.

In future studies, other factors influencing immersion like the quality of visual information, the quality of communication channel and the haptic interface have to be investigated. In the current study we focused on task primitives. As a next logical step, complex and realistic procedures should be addressed and user experience evaluations will be performed involving healthcare staff and domain experts.

\section{ACKNOWLEDGMENT}

Authors would like to thank the support received from Hospital Universitario La Paz and Hospital Carlos III (Madrid), which have collaborated to define the medical devices to be used in the experiments. Work developed by UPM researchers have been partially supported by the Spanish MINECO under ExoFlex project (DPI2015-68842R).

\section{REFERENCES}

[1] G. Stollnberger, C. Moser, E. Beck, C. Zenz, M. Tscheligi, D. Szczesniak-Stanczyk, M. Janowski, W. Brzozowski, R. L. Blaszczyk, M. Mazur, and M. Wysokinski, "Robotic systems in health care," in Proceedings of the 7th Int. Conf. on Human System Interaction, 2014, pp. 276-281.

[2] K. Doelling, J. Shin, and D. O. Popa, "Service robotics for the home: a state of the art review," in Int. Conf. on Pervasive Technologies Related to Assistive Environments. ACM, 2014, p. 35.

[3] T. L. Chen, C.-H. A. King, A. L. Thomaz, and C. C. Kemp, "An investigation of responses to robot-initiated touch in a nursing context," Int. Journal of Social Robotics, vol. 6, no. 1, pp. 141-161, 2014.
[4] Barrett Medical, "Proficio," http://www.barrettmedical.com/, accessed: 2016-02-19.

[5] J. Hu, A. Edsinger, Y.-J. Lim, N. Donaldson, M. Solano, A. Solochek, and R. Marchessault, "An advanced medical robotic system augmenting healthcare capabilities-robotic nursing assistant," in Int. Conf. on Robotics and Automation. IEEE, 2011, pp. 6264-6269.

[6] A. I. Chen, M. L. Balter, T. J. Maguire, and M. L. Yarmush, "Realtime needle steering in response to rolling vein deformation by a 9dof image-guided autonomous venipuncture robot," in Int. Conf. on Intelligent Robots and Systems. IEEE, 2015, pp. 2633-2638.

[7] J. Y. Chen, E. C. Haas, and M. J. Barnes, "Human performance issues and user interface design for teleoperated robots," Trans. on Systems, Man, and Cybernetics, Part C (Applications and Reviews), vol. 37, no. 6, pp. 1231-1245, 2007.

[8] V. Nitsch and B. Färber, "A meta-analysis of the effects of haptic interfaces on task performance with teleoperation systems," Trans. on Haptics, vol. 6, no. 4, pp. 387-398, 2013.

[9] C. G. Cao, M. Zhou, D. B. Jones, and S. D. Schwaitzberg, "Can surgeons think and operate with haptics at the same time?" Journal of Gastrointestinal Surgery, vol. 11, no. 11, pp. 1564-1569, 2007.

[10] L. N. Verner and A. M. Okamura, "Force \& torque feedback vs force only feedback," in EuroHaptics Conf. IEEE, 2009, pp. 406-410.

[11] A. Schneider, "Evaluation of haptic human-machine interfaces for virtual reality applications," Master's Thesis at the German Aerospace Center, 2015.

[12] R. Weller and G. Zachmann, "User performance in complex bi-manual haptic manipulation with 3 dofs vs. 6 dofs," in Haptics Symposium. IEEE, 2012, pp. 315-322.

[13] A. Sherman, M. C. Çavusoglu, and F. Tendick, "Comparison of teleoperator control architectures for palpation task," in ASME Int. Mechanical Eng. Congress and Exposition, 2000, pp. 1261-1268.

[14] K. Hastrudi-Zaad and S. Salcudean, "On the use of local force feedback for transparent teleoperation," in Int. Conf. on Robotics and Automation, vol. 3. IEEE, 1999, pp. 1863-1869.

[15] J. Artigas, R. Balachandran, C. Riecke, M. Stelzer, B. Weber, J.-H. Ryu, and A. Albu-Schaeffer, "Kontur-2: force-feedback teleoperation from the international space station," in Int. Conf. on Robotics and Automation. IEEE, 2016, pp. 1166-1173.

[16] B. Weber, A. Hellings, A. Tobergte, and M. Lohmann, "Human performance and workload evaluation of input modalities for telesurgery," GfA Press, 2013.

[17] J. Prieler, "Sensomotorische Koordination (Version 23.00)," 2002.

[18] S. S. Shapiro and M. B. Wilk, "An analysis of variance test for normality (complete samples)," Biometrika, vol. 52, no. 3/4, pp. 591$611,1965$.

[19] D. A. Lawrence, "Stability and transparency in bilateral teleoperation," Trans. on Robotics and Automation, vol. 9, no. 5, pp. 624-637, 1993.

[20] T. H. Massie and J. K. Salisbury, "The phantom haptic interface: A device for probing virtual objects," in Symposium on haptic interfaces for virtual environment and teleoperator systems, vol. 55, no. 1. Chicago, IL, 1994, pp. 295-300.

[21] P. Berkelman and J. Ma, "A compact modular teleoperated robot system for laparoscopic surgery," The Int. journal of robotics research, 2009.

[22] S. Ullrich and T. Kuhlen, "Haptic palpation for medical simulation in virtual environments," Trans. on Visualization and Computer Graphics, vol. 18, no. 4, pp. 617-625, 2012.

[23] F. Conti, J. Park, and O. Khatib, "Interface design and control strategies for a robot assisted ultrasonic examination system," in Experimental Robotics. Springer, 2014, pp. 97-113.

[24] G. P. Mylonas, P. Giataganas, M. Chaudery, V. Vitiello, A. Darzi, and G.-Z. Yang, "Autonomous efast ultrasound scanning by a robotic manipulator using learning from demonstrations," in Int. Conf. on Intelligent Robots and Systems. IEEE, 2013, pp. 3251-3256.

[25] O. Weede, H. Mönnich, B. Müller, and H. Wörn, "An intelligent and autonomous endoscopic guidance system for minimally invasive surgery," in Int. Conf. on Robotics and Automation. IEEE, 2011, pp. $5762-5768$.

[26] M. A. Vidulich and P. S. Tsang, "Absolute magnitude estimation and relative judgement approaches to subjective workload assessment," in Proceedings of the Human Factors and Ergonomics Society Annual Meeting, vol. 31, no. 9. SAGE Publications, 1987, pp. 1057-1061. 